\title{
STRUCTURE OF PARABOLICALLY INDUCED MODULES FOR AFFINE KAC-MOODY ALGEBRAS
}

\author{
VYACHESLAV FUTORNY AND IRYNA KASHUBA
}

\author{
Dedicated to Efim Zelmanov in the occasion of his 60th birthday
}

\begin{abstract}
The main result of the paper establishes the irreducibility of a large family of nonzero central charge induced modules over Affine Lie algebras for any non standard parabolic subalgebra. It generalizes all previously known partial results and provides a a construction of many new irreducible modules.
\end{abstract}

\section{INTRODUCTION}

Let $\mathfrak{G}$ be an affine Kac-Moody algebra with a 1-dimensional center $Z=\mathbb{C} c$ and a fixed Cartan subalgebra.

The main problem in the representation theory of affine Kac-Moody algebras is a classification of all irreducible weight representations. Such classification is known in various subcategories of weight modules, e.g. in the category $\mathcal{O}$, in its generalizations [F1, [C1, [FS], in the category of modules with finite dimensional weight multiplicities and nonzero central charge [FT]. An important tool in the construction of representations of affine Lie algebras is a parabolic induction. The conjecture ([F2], Conjecture 8.1) indicates that induced modules are construction devices for irreducible weight modules. This conjecture is known to be true for $A_{1}^{(1)}$ ([F4], Proposition 6.3), for $A_{2}^{(2)},[\mathrm{Bu}]$ and for all affine Lie algebras in the case of modules with finite-dimensional weight spaces [FT], [DG].

Simplest case of parabolic induction corresponds to the induction from Borel subalgebras. Standard examples of Borel subalgebras arise from taking partitions of the root system. For affine algebras there is always a finite number of conjugacy classes by the Weyl group of such partitions and corresponding Borel subalgebras. Verma type modules induced from these Borel subalgebras were first studied and classified by Jakobsen and Kac [JK1, JK2, and by Futorny [F1, F3], and were further developed in [C1], [FS], [F2], [F4] and references therein. We will consider a more general definition of a Borel subalgebra (see below).

Nontrivial (different from Borel) parabolic subalgebras are divided into two groups, those with finite dimensional Levi subalgebras and those with infinite dimensional one. In this paper we are interested in the second case. The simplest non trivial example is given by a parabolic subalgebra whose Levi factor is the Heisenberg subalgebra together with Cartan subalgebra. Corresponding induced modules were studied in recent papers FK2] and [BBFK]. It was shown that any irreducible $\mathbb{Z}$-graded module over the Heisenberg subalgebra with a nonzero central charge induces the irreducible $\mathfrak{G}$-module. In [FK1] a similar reduction theorem was shown for pseudo parabolic subalgebras. These parabolic 
subalgebras give a particular class of non-solvable parabolic subalgebra of $\mathfrak{G}$ with infinite dimensional Levi factor. The main results of [FK1] states that in this case the parabolic induction preserves irreducibility if the central charge is nonzero. The technique used in the proofs in [FK1] and [FK2] are different and somewhat complementary.

The main purpose of the present paper is to show that in the affine setting both these cases of parabolic induction (and hence all known cases) can be extended to a more general result for modules with nonzero central charge.

For any Lie algebra $\mathfrak{a}$ we denote by $U(\mathfrak{a})$ the universal enveloping algebra of $\mathfrak{a}$.

Denote by $G$ the Heisenberg subalgebra of $\mathfrak{G}$ generated by all imaginary root subspaces of $\mathfrak{G}$. Let $\mathcal{P} \subset \mathfrak{G}$ be a parabolic subalgebra of $\mathfrak{G}$ such that $\mathcal{P}=\mathfrak{l} \oplus \mathfrak{n}$ is a Levi decomposition and $\mathfrak{l}$ is an infinite dimensional Levi factor. Denote by $\mathfrak{l}^{0}$ the Lie subalgebra of $\mathfrak{l}$ generated by all its real root subspaces and $\mathfrak{H}$. Let $G(\mathfrak{l})$ be a subalgebra of $\mathfrak{l}^{0}$ spanned by its imaginary root subspaces. Then $\mathfrak{l}=\mathfrak{l}^{0}+G_{\mathfrak{l}}$ where $G_{\mathfrak{l}} \subset G$ is the orthogonal complement of $G(\mathfrak{l})$ in $G$ with respect to the Killing form, that is $G=G(\mathfrak{l})+G_{\mathfrak{l}},\left[G_{\mathfrak{l}}, \mathfrak{l}^{0}\right]=0$ and $\mathfrak{l}^{0} \cap G_{\mathfrak{l}}=\mathbb{C} c$.

For a Lie algebra $\mathfrak{a}$ containing the Cartan subalgebra $\mathfrak{H}$ we say that a module $V$ is a weight module if $V=\oplus_{\mu \in \mathfrak{H}^{*}} V_{\mu}$, where

$$
V_{\mu}=\{v \in V \mid h v=\mu(h) v, \forall h \in \mathfrak{H}\} .
$$

We denote by $\mathcal{W}_{\mathfrak{G}}$ (respectively, $\mathcal{W}_{\mathfrak{l}}$ ) the category of weight $\mathfrak{G}$-modules (respectively, $\mathfrak{l}$ modules) with respect to the common Cartan subalgebra $\mathfrak{H}$ of both $\mathfrak{G}$ and $\mathfrak{l}$. We say that a module $V$ from either category has a nonzero central charge if the central element of $\mathfrak{G}$ acts on $V$ as a nonzero scalar. If $N \in \mathcal{W}_{\mathfrak{l}}$ then denote by $\operatorname{ind}_{N}(\mathcal{P}, \mathfrak{G})$ the induced $\mathfrak{G}$-module $U(\mathfrak{G}) \otimes_{U(\mathcal{P})} N$, where $\mathfrak{n} N=0$. This defines a functor ind $(\mathcal{P}, \mathfrak{G})$ from the category $\mathcal{W}_{\mathfrak{l}}$ to the category $\mathcal{W}_{\mathfrak{G}}$. Denote by $\widetilde{\mathcal{W}}_{\mathfrak{l}}$ the full subcategory of $\mathcal{W}_{\mathfrak{r}}$ consisting of those modules on which the central element $c$ acts injectively and let $\widetilde{\operatorname{ind}}(\mathcal{P}, \mathfrak{G})$ be the restriction of ind $(\mathcal{P}, \mathfrak{G})$ onto $\widetilde{\mathcal{W}_{l}}$.

Since $\mathfrak{l}$ is a sum of two commuting Lie subalgebras $\mathfrak{l}^{0}$ and $G_{\mathfrak{l}}$ then a natural way to construct irreducible modules in $\widetilde{\mathcal{W}}_{\mathfrak{l}}$ is to take a tensor product of an irreducible weight module $L$ over $\mathfrak{l}^{0}$ with a $\mathbb{Z}$-graded irreducible module $T$ over $G_{\mathfrak{l}}$ with the same scalar action of $c$. We will call such modules tensor.

For any positive integer $k$, denote $\mathfrak{G}_{k}=\mathfrak{G}_{k \delta} \oplus \mathbb{C} c \oplus \mathfrak{G}_{-k \delta}$ (see notations in the next section). We say that a $\mathfrak{G}_{k}$-module $S$ is $U\left(\mathfrak{G}_{k \delta}\right)$-surjective (respectively $U\left(\mathfrak{G}_{-k \delta}\right)$-surjective) if for any two elements $s_{1}, s_{2} \in S$ there exist $s \in S$ and $u_{1}, u_{2} \in U\left(\mathfrak{G}_{k \delta}\right)$ (respectively,

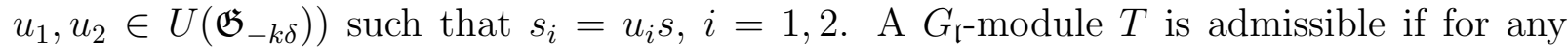
positive integer $k$, any cyclic $\mathfrak{G}_{k}$-submodule $T^{\prime} \subset T$ is $U\left(\mathfrak{G}_{k \delta}\right)$-surjective or $U\left(\mathfrak{G}_{-k \delta}\right)$ surjective.

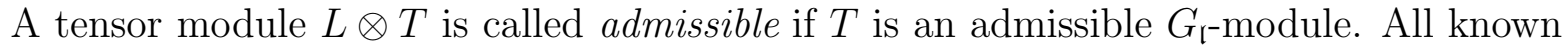
to us examples of irreducible l-modules are admissible tensor modules. On the other hand we do not have sufficient evidence to expect that admissible tensor modules exhaust all irreducible modules in $\widetilde{\mathcal{W}_{l}}$ or even tensor modules.

Our main result is the following theorem: 
Theorem 1. Let $\mathcal{P} \subset \mathfrak{G}$ be a parabolic subalgebra of $\mathfrak{G}$ such that $\mathcal{P}=\mathfrak{l} \oplus \mathfrak{n}$ is a Levi decomposition and $\mathfrak{l}$ is infinite dimensional Levi factor. Then $\widetilde{\operatorname{ind}_{N}}(\mathcal{P}, \mathfrak{G})$ is an irreducible $\mathfrak{G}$-module for any irreducible admissible tensor module $N$ from $\widetilde{\mathcal{W}}_{\mathfrak{l}}$.

We see that this result is quite general allowing one to induce from an arbitrary irreducible admissible tensor $\mathfrak{l}$-module with nonzero central charge and to construct many new irreducible modules over $\mathfrak{G}$. This generalises the results from [FK1], [FK2], BBFK]. In fact, it is possible to go beyond the category of weight modules but some grading is required in order to apply the same technique. Note that the proof of Theorem 1 in [FK2] is only valid for admissible $G$-modules, it is a particular case of the theorem above.

All results in the paper hold for both untwisted and twisted affine Lie algebras.

\section{Preliminaries}

We address to $[\mathrm{K}]$ for the basics of the Kac-Moody theory. The affine Lie algebra $\mathfrak{G}$ has the root decomposition

$$
\mathfrak{G}=\mathfrak{H} \oplus\left(\oplus_{\alpha \in \Delta} \mathfrak{G}_{\alpha}\right),
$$

where $\mathfrak{G}_{\alpha}=\{x \in \mathfrak{G} \mid[h, x]=\alpha(h) x$ for every $h \in \mathfrak{H}\}$ and $\Delta$ is the root system of $\mathfrak{G}$. Let $\pi$ be a fixed basis of the root system $\Delta$. Then the root system $\Delta$ of has a natural partition into positive and negative roots with respect to $\pi, \Delta_{+}$and $\Delta_{-}$respectively. Let $\delta \in \Delta_{+}(\pi)$ be the indivisible imaginary root. Then the set of all imaginary roots is $\Delta^{i m}=\{k \delta \mid k \in \mathbb{Z} \backslash\{0\}\}$. Let $G=\oplus_{k \in \mathbb{Z} \backslash\{0\}} \mathfrak{G}_{k \delta} \oplus \mathbb{C} c$, a Heisenberg subalgebra of $\mathfrak{G}$. Then $G$ has a triangular decomposition $G=G_{-} \oplus \mathbb{C} c \oplus G_{+}$, where $G_{ \pm}=\oplus_{k>0} \mathfrak{G}_{ \pm k \delta}$.

Denote by $\mathfrak{g}$ the underlined simple finite dimensional Lie algebra that has $\left\{\alpha_{1}, \ldots, \alpha_{N}\right\} \subset$ $\pi$ as set of simple roots and $\dot{\Delta}=\dot{\Delta}_{+} \cup \dot{\Delta}_{-}$be a decomposition of the root system $\dot{\Delta}$ of $\mathfrak{g}$ into positive and negative to this set of simple roots. When there are roots of two lengths in $\mathfrak{g}$, we set $\dot{\Delta}_{l}$ and $\dot{\Delta}_{s}$ to be the long and short roots in $\dot{\Delta}$ respectively. The real roots $\Delta^{\text {re }}$ of $\Delta$ can be described as follows: $\Delta^{\text {re }}=S \cup-S$, where

$$
S=\left\{\begin{array}{cr}
\left\{\alpha+n \delta \mid \alpha \in \dot{\Delta}_{+}, n \in \mathbb{Z}\right\}, & \text { if } r=1 \text { (the untwisted case), } \\
\left\{\alpha+n \delta \mid \alpha \in\left(\dot{\Delta}_{s}\right)_{+}, n \in \mathbb{Z}\right\} \cup\left\{\alpha+n r \delta \mid \alpha \in\left(\dot{\Delta}_{l}\right)_{+}, n \in \mathbb{Z}\right\} & \text { if } r=2,3 \text { and } \\
\left\{\alpha+n \delta \mid \alpha \in\left(\dot{\Delta}_{s}\right)_{+}, n \in \mathbb{Z}\right\} \cup\left\{\alpha+2 n \delta \mid \alpha \in\left(\dot{\Delta}_{l}\right)_{+}, n \in \mathbb{Z}\right\} & \text { not } \mathrm{A}_{2 \ell}^{(2)} \text { type, } \\
\cup\left\{\frac{1}{2}(\alpha+(2 n-1) \delta) \mid \alpha \in\left(\dot{\Delta}_{l}\right)_{+}, n \in \mathbb{Z}\right\} & \text { if } \mathrm{A}_{2 \ell}^{(2)} \text { type. }
\end{array}\right.
$$

\section{Parabolic induCtion}

In this section we consider our main tool of constructing new modules - parabolic induction. We start with the discussion of the Borel subalgebras.

3.1. Borel subalgebras. A subalgebra $\mathfrak{B} \subset \mathfrak{G}$ is called a Borel subalgebra if it contains $\mathfrak{H}$ (and hence has a root decomposition) and there exists an automorphism $\sigma$ of $\mathfrak{G}$ satisfying

- $\sigma(\mathfrak{H})=\mathfrak{H}$; 
- $\mathfrak{B}+\sigma(\mathfrak{B})=\mathfrak{G}$

- $\mathfrak{B} \cap \sigma(\mathfrak{B})=\mathfrak{H}$.

Note that this definition is more general than the usual definition of Borel subalgebras associated with closed partitions of root systems [F1], [DFG].

Consider a subset $P \subset \Delta$ such that $P \cap(-P)=\emptyset$ and $P \cup(-P)=\Delta$. Denote by $\mathfrak{B}_{P}$ a Lie subalgebra of $\mathfrak{G}$ generated by $\mathfrak{H}$ and the root spaces $\mathfrak{G}_{\alpha}$ with $\alpha \in P$. We will say that $P$ is a quasi partition of $\Delta$ if for any root $\alpha$ of $\mathfrak{B}_{P}$ we have $\alpha \in P$. Note that in a contrast with a usual concept of a partition of the root systems [F1] we do not require $P$ to be a closed subset with respect to the sum of roots, that is whenever $\alpha$ and $\beta$ are in $P$ and $\alpha+\beta$ is a root, then $\alpha+\beta \in P$. A subset of all real roots of any quase partition is closed with respect to the sum of the roots while it is not necessary for imaginary roots (cf. [BBFK]). Clearly, $\mathfrak{B}_{P}$ is a Borel subalgebra for any quase partition $P$. These subalgebras are a main source of examples of Borel subalgebras, though they do not exhaust all of them as shown in the following remark.

Remark 1. In each subspace $G_{k \delta}$ we can choose a commuting basis $x_{k 1}, \ldots, x_{k s_{k}}$ such that $\left[x_{k i}, x_{-k j}\right]=\delta_{i j}$ for any $k$ and all $i, j$. We can define a triangular decomposition of $G$ : $G=G_{1} \oplus \mathbb{C} c \oplus G_{-1}$, where for each nonzero $k, G_{k \delta} \subset G_{i}$ implies $G_{-k \delta} \subset G_{-i}, i=1,-1$. On the other hand we can obtain a more general triangular decomposition by splitting for every $k, x_{k j}$ and $x_{-k j}$ between $G_{1}$ and $G_{-1}$ for each $j$ independently, $j=1, \ldots, s_{k}$. Each such triangular decomposition can be extended to the following decomposition of $\mathfrak{G}$. Denote by $B_{ \pm}$a Lie subalgebra of $\mathfrak{G}$ generated by all root spaces $\mathfrak{G}_{ \pm \beta}, \beta \in\left\{\alpha+k \delta \mid \alpha \in \dot{\Delta}_{+}, k \in \mathbb{Z}\right\}$ and $G_{ \pm 1}$. Then

$$
\mathfrak{G}=B_{-} \oplus \mathfrak{H} \oplus B_{+}
$$

and $B=\mathfrak{H} \oplus B_{+}$is a Borel subalgebra of $\mathfrak{G}$. In particular, if $x_{k j}$ are in the same $G_{i}$ for all positive $k$ and all $j=1, \ldots, s_{k}$ then $B$ corresponds to a partition of the root system. If for some positive $k \neq m, x_{k j}$ and $x_{m r}$ belong to different $G_{i}$ but for any $k, x_{k j}$ are in the same $G_{i}$ for all $j=1, \ldots, s_{k}$, then $B$ corresponds to a quase partition of the root system (cf. $\mathfrak{B}_{\text {nat }}^{\varphi}$ below).

Classification of partitions of the root system and corresponding Borel subalgebras was obtained in [JK1] and [F1]. Classification of quasi partitions follows from [BBFK]. Finally, the classification of all Borel subalgebras defined above can easily be deduced from Remark 1.

There are two extreme Borel subalgebras, the standard Borel subalgebra which corresponds to the partition $P=\Delta_{+}$and the natural Borel subalgebra $\mathfrak{B}_{\text {nat }}=\mathfrak{B}_{P}$ which corresponds to the partition

$$
P_{\text {nat }}=\left\{\alpha+k \delta \mid \alpha \in \dot{\Delta}_{+}, k \in \mathbb{Z}\right\} \cup\{n \delta \mid n>0\} .
$$

These Borel subalgebras are not conjugated by the Weyl group. For other conjugacy classes of Borel subalgebras by the Weyl group see [F1]. We will be interested mainly in $\mathfrak{B}_{\text {nat }}$ in this paper. Starting from this Borel subalgebra one can construct a family of twisted 
subalgebras as in [BBFK]. For a function $\varphi: \mathbb{N} \rightarrow\{ \pm\}$, set

$$
P^{\varphi}=\left\{\alpha+k \delta \mid \alpha \in \dot{\Delta}_{+}, k \in \mathbb{Z}\right\} \cup\{n \delta \mid n \in \mathbb{N}, \varphi(n)=+\} \cup\{-m \delta \mid m \in \mathbb{N}, \varphi(m)=-\}
$$

and $\mathfrak{B}_{\text {nat }}^{\varphi}=\mathfrak{B}_{P \varphi}$. Further examples of Borel subalgebras can be obtained by combining $\varphi$-twisting with the procedure described in Remark 1 .

3.2. Parabolic subalgebras. A subalgebra $\mathcal{P} \subset \mathfrak{G}$ is called a parabolic subalgebra if it contains a Borel subalgebra. There are essentially two types of parabolic subalgebras: those containing the standard Borel and those containing one of the twisted Borel subalgebras $\mathfrak{B}_{\text {nat. }}^{\varphi}$. We call them type I and type II parabolic subalgebras respectively (cf. [F2] for details). We address in the paper the parabolic subalgebras of type II. Even though all the results of this paper are valid for all parabolic subalgebras of type $I I$ we will assume for simplicity that a fixed parabolic subalgebra $\mathcal{P}$ of type $I I$ contains $\mathfrak{B}_{\text {nat }}$. We will describe all such $\mathcal{P}$ 's which contain properly $\mathfrak{B}_{\text {nat }}$ (cf. also Proposition 3.3, FK1]).

Let $N$ be the rank of the underlined simple finite dimensional Lie algebra $\dot{\mathfrak{g}}$ and $\pi_{0}=$ $\left\{\alpha_{1}, \ldots, \alpha_{N}\right\} \subset \pi$ the set of simple roots of $\dot{\mathfrak{g}}$. Set $I=\{1, \ldots, N\}$ and choose any proper subset $J \subset I$. Let $\pi^{J}=\left\{\alpha_{j} \in \pi \mid j \in J\right\}$. Denote by $\dot{\Delta}^{J}$ the finite root system generated by the roots in $\pi^{J}\left(\dot{\Delta}^{J}=\emptyset\right.$ if $\left.J=\emptyset\right)$. Now consider the affinization

$$
\Delta^{J}=\left\{\alpha+n \delta \in \Delta \mid \alpha \in \dot{\Delta}^{J}, n \in \mathbb{Z}\right\} \cup\{n \delta \mid n \in \mathbb{Z} \backslash\{0\}\},
$$

of $\dot{\Delta}^{J}$ (in $\Delta$ ).

Define $\mathcal{P}_{J}=\mathfrak{B}_{\text {nat }}+\sum_{\alpha \in \Delta^{J}} \mathfrak{G}_{\alpha}$. This is the parabolic subalgebra of type II associated with $J$. Again it admits modifications as in Remark 1 but we will not consider it though the main statement remains valid also in this case. If $J=\emptyset$ then $\mathcal{P}_{\emptyset}=\mathfrak{B}_{\text {nat }}+G$. The parabolic subalgebra $\mathcal{P}_{J}$ has the Levi decomposition $\mathcal{P}_{J}=\mathfrak{l}_{J} \oplus \mathfrak{n}_{J}$ where $\mathfrak{l}_{J}$ is a Lie subalgebra generated by $\mathfrak{G}_{\alpha}$ with $\alpha \in \Delta^{J}$ and $\mathfrak{n}_{J}=\sum_{\alpha \in P_{\text {nat }} \backslash \Delta^{J}} \mathfrak{G}_{\alpha}$. Note that $\mathfrak{l}_{\emptyset}=G+\mathfrak{H}$.

Denote $\mathfrak{n}_{\bar{J}}=\sum_{\alpha \in-P_{\text {nat }} \backslash \Delta^{J}} \mathfrak{G}_{\alpha}$. Then we have the following decomposition of $\mathfrak{G}: \mathfrak{G}=$ $\mathfrak{n}_{\bar{J}} \oplus \mathcal{P}_{J}=\mathfrak{n}_{\bar{J}} \oplus \mathfrak{l}_{J} \oplus \mathfrak{n}_{J}$. Note that this may not be a triangular decomposition in the classical sense of [MP]. Nevertheless this decomposition allows us to construct families of induced modules.

We say that a subset $S \subseteq J$ is connected if the Coxeter-Dynkin diagram associated to the simple roots $\alpha_{i}, i \in S$ is connected. Then $J=\cup_{t \in T} S_{t}$ where $S_{t}$ 's are connected components of the Coxeter-Dynkin diagram associated to $J$. Each subset $S_{t}$ gives rise to an affine root subsystem of $\Delta^{J}$ which generates an affine Lie subalgebra $\mathfrak{l}_{J}\left(S_{t}\right) \subset \mathfrak{l}_{J}$. Also denote by $G_{J} \subset G$ the orthogonal completion (with respect to the Killing form) of the Heisenberg subalgebra $G\left(\mathfrak{l}_{J}\right)$ of $\mathfrak{l}_{J}$. Then $G=G_{J}+G\left(\mathfrak{l}_{J}\right),\left[G_{J}, \mathfrak{l}_{J}\right]=0$ and we have

$$
\mathfrak{l}_{J}=\sum_{t \in T} \mathfrak{l}_{J}\left(S_{t}\right)+G_{J}+\mathfrak{H} \text {. }
$$

3.3. Induced modules. We will assume that $\mathcal{P}_{J}$ is the parabolic subalgebra of type II associated with fixed subset $J, \mathcal{P}_{J}=\mathfrak{l}_{J} \oplus \mathfrak{n}_{J}$. Let $N$ be a weight (with respect to $\mathfrak{H}$ ) module over $\mathcal{P}_{J}$ with a trivial action of $\mathfrak{n}_{J}$. Define the induced $\mathfrak{G}$-module

$$
M_{J}(N)=\operatorname{ind}_{N}\left(\mathcal{P}_{J}, \mathfrak{G}\right) .
$$


This is the generalized Imaginary Verma module associated with $J$ and $N$.

If $N$ is irreducible then $M_{J}(N)$ has a unique irreducible quotient $L_{J}(N)$. Basis properties of modules $M_{J}(N)$ are collected in the following proposition. Details of the proofs can be found in [F2].

Proposition 1. Let $J \subseteq I$ and $N$ irreducible weight $\mathfrak{l}_{J}$-module. Then $M_{J}(N)$ has the following properties.

(i) The module $M_{J}(N)$ is a free $U\left(\mathfrak{n}_{\bar{J}}\right)$-module.

(ii) Let $V$ be a nonzero $\mathfrak{G}$-module generated by a weight vector $v$ such that $\mathfrak{n}_{J} v=0$. Set $N=U\left(\mathfrak{l}_{J}\right) v$. Then there exists a unique surjective homomorphism $\psi: M_{J}(N) \mapsto V$ such that $\psi(1 \otimes v)=v$. If $V$ is irreducible then $N$ is irreducible $\mathfrak{l}_{J}$-module and $V \simeq L_{J}(N)$.

(iii) $M_{J}(N)$ is a weight module. Moreover, $0<\operatorname{dim} M_{J}(N)_{\mu}<\infty$ if and only if $\mu$ is a weight of $N$ and $0<\operatorname{dim} N_{\mu}<\infty$.

(iv) If $N$ is irreducible $\mathfrak{l}$-module then the subspace of $\mathfrak{n}_{J}$-invariants in $L_{J}(N)$ is the class of $1 \otimes N$.

(v) If $J=\emptyset$ and $N$ is a highest weight $G$-module generated by a weight vector $v$ such that $h v=\lambda(h) v$ for any $h \in \mathfrak{H}$ and some $\lambda \in \mathfrak{H}^{*}$ and $\mathfrak{n}_{\emptyset} v=0$ then $M_{\emptyset}(N)$ is an Imaginary Verma type module $M(\lambda)$ generated by an eigenvector for the natural Borel subalgebra $\mathfrak{B}_{\text {nat }}$.

Remark 2. (i) Generalized loop modules considered in [FK2] and, in particular, $\varphi$ Imaginary Verma modules [BBFK] are partial cases of modules $M_{\emptyset}(N)$ when $N$ is irreducible G-module;

(ii) Pseudo-parabolic induction considered in [FK1] is a particular case of modules $M_{J}(N)$ where $G_{J} N=0$.

Set $M_{J}^{t}(N):=1 \otimes N$. This is the "top" part of $M_{J}^{t}(N)$ which generates $M_{J}(N)$.

3.3.1. Tensor $\mathfrak{l}_{J}$-modules. Denote $\mathfrak{l}_{J}^{0}=\sum_{t \in T} \mathfrak{l}_{J}\left(S_{t}\right)+\mathfrak{H}$. Hence $\mathfrak{l}_{J}=\mathfrak{l}_{J}^{0}+G_{J}$ and $\left[\mathfrak{l}_{J}^{0}, G_{J}\right]=$ 0 . If $V$ is an irreducible weight $\mathfrak{l}_{J}^{0}$-module and $W$ is an irreducible $\mathbb{Z}$-graded $G_{J}$-module with the same central charge then $V \otimes W$ is naturally an $\mathfrak{l}_{J}$-module, a tensor module. If the central charge is $a \in \mathbb{C}$ then $V \otimes W$ is a module over the tensor product $U\left(\mathfrak{l}_{J}^{0}\right) /(c-$ a) $\otimes U\left(G_{J}\right) /(c-a)$.

In the extreme case when $J=\emptyset$ we have $\mathfrak{l}_{J}^{0}=\mathfrak{H}$ and $G_{J}=G$. Hence $V$ is a 1dimensional space and $W$ is a $\mathbb{Z}$-graded $G$-module. Parabolic induction functor from $G$ to $\mathfrak{G}$ was considered in [FK2].

Suppose now that $J \neq \emptyset$. Let $N$ be an irreducible weight $\mathfrak{l}_{J}$-module with a nonzero central charge $a$. Assume that for each positive integer $k$ either $\left(G_{J}\right)_{k \delta}$ or $\left(G_{J}\right)_{-k \delta}$ acts trivially on $N$. Such $\mathfrak{l}_{J}$-modules were considered in [FK1] when inducing from pseudo parabolic subalgebras. We will show that any such irreducible module is a tensor module. Without loss of generality we assume that $\left(G_{J}\right)_{k \delta} N=0$ for all positive integer $k$.

Proposition 2. $N$ is a tensor $\mathfrak{l}_{J}$-module, that is $N \simeq V \otimes W$ where $V$ is irreducible weight $\mathfrak{l}_{J}^{0}$-module and $W$ is irreducible $\mathbb{Z}$-graded $G_{J}$-module. 
STRUCTURE OF PARABOLICALLY INDUCED MODULES FOR AFFINE KAC-MOODY ALGEBRAS 7

Proof. Choose nonzero element $v \in N$. Denote $V=U\left(\mathfrak{l}_{J}^{0}\right) v$ and $W=U\left(G_{J}\right) v$. It is standard that $W$ is irreducible $G_{J}$-module since the central charge is nonzero and $G_{J}^{+} v=0$. Suppose $V$ is not irreducible and $V^{\prime}$ is a nonzero proper $\mathfrak{l}_{J^{-}}^{0}$-submodule. Take any nonzero $v^{\prime} \in V^{\prime}$. Then $v^{\prime}=x v$ for some $x \in U\left(\mathfrak{l}_{J}^{0}\right)$. Since $N$ is irreducible there exists $y \in U\left(G_{J}\right)$ such that $y x v=v$. Moreover, we can assume that $y \in U\left(G_{J}^{-}\right)$. But the $G_{J}$-module $U\left(G_{J}\right) x v$ is irreducible. Hence, there exists $y^{\prime} \in U\left(G_{J}^{+}\right)$such that $y^{\prime} y x v=y^{\prime} v=x v$. But $y^{\prime} v=0$, thus $v^{\prime}=x v=0$ which is a contradiction. We conclude that $V$ is $\mathfrak{l}_{J}^{0}$-module. Then $V \otimes W$ is irreducible $\mathfrak{l}_{J}$-module. Consider a map $f: V \otimes W \rightarrow N$, sending $x v \otimes y v$ to $x y v$ which is clearly a homomorphism since $\mathfrak{l}_{J}^{0}$ and $G_{J}$ commute. We immediately see that $f$ is surjective since $N$ is irreducible. If $x y v=0$ then choose $y^{\prime}$ as above. We have $0=y^{\prime} y x v=x v$ and $f$ is injective. Therefore, $N \simeq V \otimes W$.

These are all known cases when parabolic induction preserves irreducibility and in all cases we induce from certain tensor modules. Combining techniques from [FK1] and [FK2] we will extend the proof to all tensor modules.

\section{Irreducibility of Generalized Imaginary Verma modules}

In this section we prove our main result by finding conditions for a module $M_{J}(N)$ to be irreducible.

Let $\mathcal{P}_{J}=\mathfrak{l}_{J} \oplus \mathfrak{n}_{J}$ and $N \in W_{\mathfrak{l}}$. Denote by $T_{J}(N)$ the subspace of $\mathfrak{n}_{J}$-invariants in $M_{J}(N)$, that is $v \in T_{J}(N)$ if and only if $\mathfrak{n}_{J} v=0$.

Theorem 2. If $U$ is an irreducible admissible tensor module in $\widetilde{W}_{\mathfrak{r}}$ then $T_{J}(U)=M_{J}^{t}(U)$.

The proof of Theorem 2 combines the proofs of Theorem 2 from [FK2] and Theorem 3.1 from [FK1] where particular cases of parabolic induction were considered.

For any subset $\omega \subset I$, let $Q_{ \pm}^{\omega}$ denote a semigroup of $\mathfrak{H}^{*}$ generated by $\pm \alpha_{i}, i \in \omega$. Set $Q^{J}=\oplus_{j \in J} \mathbb{Z} \alpha_{j} \oplus \mathbb{Z} \delta$ and $Q_{ \pm}=Q_{ \pm}^{I}$. Then $Q_{ \pm}^{J}=Q^{J} \cap Q_{ \pm}$.

Let $\alpha \in Q_{-}^{J}$ and $\alpha=-\sum_{j \in \omega} k_{j} \alpha_{j}$, where each $k_{j}$ is in $\mathbb{Z}_{\geq 0}$. We set ht ${ }_{J}(\alpha)=\sum_{j=1}^{n} k_{j}$, the J-height of $\alpha$.

Let $v \in M_{J}(N)$ be a nonzero weight element. Then

$$
v=\sum_{i \in R} u_{i} v_{i}
$$

for some finite set $R$, where $u_{i} \in U\left(\mathfrak{n}_{\bar{J}}\right)$ are linearly independent homogeneous elements, $v_{i} \in N, i \in R$. Since $v$ is a weight vector then each $u_{i}$ is a homogeneous element of $U\left(\mathfrak{n}_{\bar{J}}\right)$. Its homogeneous degree is an element of $Q_{-}^{\bar{J}}+Q^{J}+\mathbb{Z} \delta$. Suppose that $u_{i}$ has homogeneous degree

$$
\varphi_{i}=-\sum_{j \in \bar{J}} k_{i j} \alpha_{j}+\sum_{j \in J} l_{i j} \alpha_{j}+m_{i} \delta,
$$

where $k_{i j} \in \mathbb{Z}_{\geq 0}$ not all zeros, $l_{i j} \in \mathbb{Z}$ and $m_{i} \in \mathbb{Z}$. Since $v$ is a weight vector then all $\varphi_{i}$ have the same J-height. We will call it the J-height of $v$ and denote $\mathrm{ht}_{J}(v)$. 
Lemma 1. Suppose $U \in \widetilde{W}_{\mathfrak{l}}$ and $v \in M_{J}(U)$ a nonzero weight element such that $\mathrm{ht}_{J}(v)>1$. Then there exists $u \in U\left(\mathfrak{n}_{J}\right)$ such that $u v \neq 0$ and $\mathrm{ht}_{J}(u v)=\mathrm{ht}_{J}(v)-1$.

Proof. Let $v=\sum_{i \in R} u_{i} v_{i}$, where $u_{i} \in U\left(\mathfrak{n}_{\bar{J}}\right)$ are linearly independent homogeneous elements and $v_{i} \in U$ are nonzero elements, $i \in R$. We assume that for each $i$, $u_{i}$ has homogeneous degree $\varphi_{i}$ and all $\varphi_{i}$ have the same J-height. Then we can apply exactly the same argument as in the proof of the induction step in Lemma 5.3 in [BBFK]. We refer to [BBFK] for details.

It follows immediately from Lemma 1 that $T_{J}(N)$ can not contain nonzero elements of J-height $\mathrm{ht}_{J}(v)>1$. Indeed, any such element would generated a proper submodule whose elements would have J-heights $\geq \mathrm{ht}_{J}(v)$ which contradicts Lemma 1. Note that the proof of Lemma 1 does not work in the case when $\mathrm{ht}_{J}(v)=1$. This case requires a more delicate treatment. We consider first the case when $J=\emptyset$. This case was treated in [FK2] where the key point was Lemma 1. But the proof of this lemma is somewhat incomplete so we address the proof here in more details.

Set $\mathfrak{l}=\mathfrak{l}_{\emptyset}=G+\mathfrak{H}$.

Lemma 2. Let $W \in \widetilde{W}_{\mathfrak{l}}$ be an irreducible and admissible, $v \in W$ a nonzero element and $u_{2}, \ldots, u_{s} \in U(G)$ nonzero homogeneous elements of nonzero degrees $k_{2}, \ldots k_{s}$ respectively, that is $u_{i} \in U(G)_{k_{i} \delta}$, such that $u_{i} v \neq 0, i=2, \ldots, s, k_{i} \neq k_{j}$ if $i \neq j$. Then there exists $N \in \mathbb{Z}$ for which

$$
z_{N}=x_{N} v+\sum_{i=2}^{s} x_{N-k_{i}} u_{i} v \neq 0
$$

for any choice of nonzero $x_{k} \in G_{k \delta}$ such that $\left[x_{k}, x_{-k}\right] \neq 0$.

Proof. Since $W$ is admissible we can assume without loss of generality that $k_{i}+k_{j} \neq 0$ for all $i \neq j$. This implies $\left[u_{i}, u_{j}\right]=0$ and $\left[x_{N-k_{i}}, x_{-N-k_{j}}\right]=0$ for all $i, j$. Fix $N \in \mathbb{Z}$ and suppose that $z_{N}=z_{-N}=0$. We will assume $N$ sufficiently large. We have $x_{N} v=-\sum_{i=2}^{s} x_{N-k_{i}} u_{i} v$ and $x_{-N} v=-\sum_{i=2}^{s} x_{-N-k_{i}} u_{i} v$. Then

$$
\left[x_{N}, x_{-N}\right] v=\left(-x_{N} \sum_{i=2}^{s} x_{-N-k_{i}} u_{i}+x_{-N} \sum_{i=2}^{s} x_{N-k_{i}} u_{i}\right) v,
$$

since $\left[x_{N}, u_{i}\right]=\left[x_{-N}, u_{i}\right]=\left[x_{N-k_{i}}, x_{-N}\right]=\left[x_{N}, x_{-N-k_{i}}\right]=0$.

Fix $j=2, \ldots, s$. Then $x_{N-k_{j}} u_{j} v=-x_{N} v-\sum_{2 \leq i \neq j} x_{N-k_{i}} u_{i} v$ and $x_{-N-k_{j}} u_{j} v=-x_{-N} v-$ $\sum_{2 \leq i \neq j} x_{-N-k_{i}} u_{i} v$. We have

$$
\begin{gathered}
{\left[x_{N-k_{j}} u_{j}, x_{-N-k_{j}} u_{j}\right] v=-x_{-N} x_{N-k_{j}} u_{j} v-x_{N-k_{j}} \sum_{2 \leq i \neq j} x_{-N-k_{i}} u_{j} u_{i} v+} \\
+x_{N} x_{-N-k_{j}} u_{j} v+x_{-N-k_{j}} u_{j}\left(\sum_{2 \leq i \neq j} x_{N-k_{i}} u_{i}\right) v
\end{gathered}
$$


Since $k_{j} \neq 0,\left[x_{N-k_{j}} u_{j}, x_{-N-k_{j}} u_{j}\right]=0$ for all $j=2, \ldots, s$. We sum up all these equalities for $j=2, \ldots, s$ :

$$
\begin{gathered}
0=\sum_{j=2}^{s}\left(-x_{-N} x_{N-k_{j}}+x_{N} x_{-N-k_{j}}\right) u_{j} v-\sum_{j=2}^{s} x_{N-k_{j}}\left(\sum_{2 \leq i \neq j} x_{-N-k_{i}} u_{j} u_{i}\right) v \\
+\sum_{j=2}^{s} x_{-N-k_{j}}\left(\sum_{2 \leq i \neq j} x_{N-k_{i}} u_{j} u_{i}\right) v= \\
\sum_{j=2}^{s}\left(-x_{-N} x_{N-k_{j}}+x_{N} x_{-N-k_{j}}\right) u_{j} v=-\left[x_{N}, x_{-N}\right] v \neq 0
\end{gathered}
$$

which is a contradiction. Hence, $z_{N}=0$ or $z_{-N}=0$ which completes the proof.

Corollary 1. Suppose $J=\emptyset, W \in \widetilde{W}_{\mathfrak{l}}$ is irreducible and $v \in M_{J}(W)$ a nonzero weight element such that $\mathrm{ht}_{J}(v)=1$. Then there exists $d \in U\left(\mathfrak{n}_{J}\right)$ such that $d v \neq 0$ and $\mathrm{ht}_{J}(d v)=$ 0 .

Proof. Since $v \in M_{J}(W)$ a weight element then

$$
v=\sum_{r \in R} d_{r} w_{r}
$$

where $d_{r} \in \mathfrak{n}_{\bar{\emptyset}}$ are linearly independent, $w_{r} \in W, r \in R$. Fix $r_{0} \in R$ and assume $d_{r_{0}} \in \mathfrak{G}_{\varphi}$. Choose an integer $N$ and let $d \in \mathfrak{G}_{-\varphi+N \delta} \subset \mathfrak{n}_{\emptyset}$ be a nonzero element. Then $\left[d, d_{r_{0}}\right] \neq 0$ and we have

$$
\begin{aligned}
& d v=d d_{r_{0}} w_{r_{0}}+d \sum_{r \in R, r \neq r_{0}} d_{r} w_{r}= \\
& =\left[d, d_{r_{0}}\right] w_{r_{0}}+\sum_{r \in R, r \neq r_{0}}\left[d, d_{r}\right] w_{r} .
\end{aligned}
$$

Since $W$ is irreducible there exist $u_{r} \in U(G), r \in R$ such that $w_{r}=u_{r} w_{r_{0}}$. Note that all $u_{r}$ have different homogeneous degrees as $d_{r}$ were linearly independent. Note that $\left[d, d_{r_{0}}\right] \in G_{N \delta}$. Applying Lemma 2 for a sufficiently large $N$ we obtain $d v \neq 0$ and $\mathrm{ht}_{J}(d v)=0$.

Next we consider the case $J \neq \emptyset$.

Lemma 3. Let $U$ be a tensor module in $\widetilde{W}_{\mathfrak{l}}, U \simeq V \otimes W$ where $V$ is irreducible weight $\mathfrak{l}_{J}^{0}$-module and $W$ is irreducible $\mathbb{Z}$-graded $G_{J}$-module. Choose nonzero $v_{1}, \ldots, v_{k} \in V$ and nonzero $w_{1}, \ldots, w_{k} \in W$. Fix an integer $N>0$ and nonzero $x_{ \pm N} \in G_{ \pm N \delta}$, such that $x_{ \pm N} \notin \mathfrak{l}_{J}^{0}$ and $\left[x_{N}, x_{-N}\right] \neq 0$. Then for any $a_{i}, b_{i} \in U\left(\mathfrak{l}_{J}\right), i=2, \ldots, k$ and sufficiently large $N$ we have

$$
x_{-N}\left(v_{1} \otimes w_{1}\right)+\sum_{i=2}^{k} a_{i} v_{i} \otimes w_{i} \neq 0
$$


or

$$
x_{N}\left(v_{1} \otimes w_{1}\right)+\sum_{i=2}^{k} b_{i} v_{i} \otimes w_{i} \neq 0
$$

Proof. Assume

$$
z=x_{-N}\left(v_{1} \otimes w_{1}\right)+\sum_{i=2}^{k} a_{i} v_{i} \otimes w_{i}=0 .
$$

Suppose first that $\left[x_{ \pm N}, \mathfrak{l}_{J}^{0}\right]=0$, that is $x_{ \pm N} \in G_{J}$. Then

$$
x_{N} z=v_{1} \otimes x_{N} x_{-N} w_{1}+\sum_{i=2}^{k} a_{i} v_{i} \otimes x_{N} w_{i}=0 .
$$

If $N$ is sufficiently large then $x_{N} w_{i}$ have different gradings than $w_{1}, i=2, \ldots, k$ and thus $x_{N} x_{-N} w_{1}=0$. Similarly, if $v_{1} \otimes x_{N} w_{1}+\sum_{i=2}^{k} b_{i} v_{i} \otimes w_{i}=0$ then $x_{-N} x_{N} w_{1}=0$. But this is a contradiction. Consider now a general case. We have $x_{ \pm N}=x_{ \pm N}^{1}+x_{ \pm N}^{2}$ where $\left[x_{ \pm N}^{1}, \mathfrak{l}_{J}^{0}\right]=0$ and $x_{ \pm N}^{2} \in \mathfrak{l}_{J}^{0}$. Moreover, $x_{ \pm N}^{1} \neq 0$ since $x_{ \pm N} \notin \mathfrak{l}_{J}^{0}$ and $\left[x_{N}^{1}, x_{-N}^{1}\right] \neq 0$. Then

$$
z=v_{1} \otimes x_{-N}^{1} w_{1}+x_{-N}^{2} v_{1} \otimes w_{1}+\sum_{i=2}^{k} a_{i} v_{i} \otimes w_{i}=0
$$

and

$$
x_{N}^{1} z=v_{1} \otimes x_{N}^{1} x_{-N}^{1} w_{1}+x_{-N}^{2} v_{1} \otimes x_{N}^{1} w_{1}+\sum_{i=2}^{k} a_{i} v_{i} \otimes x_{N}^{1} w_{i}=0 .
$$

Then we proceed as in the previous case and conclude $x_{N}^{1} x_{-N}^{1} w_{1}=0$. Replacing $N$ by $-N$ we obtain $x_{-N}^{1} x_{N}^{1} w_{1}=0$ implying $w_{1}=0$ which is a contradiction. This completes the proof.

Corollary 2. Let $U \simeq V \otimes W$ be a tensor module in $\widetilde{W}_{\mathfrak{l}}$, where $V$ is irreducible weight $\mathfrak{l}_{J}^{0}$-module and $W$ is irreducible $G_{J}$-module. For a nonzero weight element $v \in M_{J}(U)$ with $\mathrm{ht}_{J}(v)=1$ there exists $u \in U\left(\mathfrak{n}_{J}\right)$ such that $u v \neq 0$ and $\mathrm{ht}_{J}(u v)=0$.

Proof. Let $v=\sum_{r \in R} d_{r}\left(v_{r} \otimes w_{r}\right)$, where $d_{r} \in \mathfrak{n}_{\bar{J}}$ are linearly independent, $v_{r} \in V, w_{r} \in W$, $r \in R$. We proceed as in the proof of Corollary 1. Fix $r_{0} \in R$ and assume $d_{r_{0}} \in \mathfrak{G}_{\varphi}$. Choose an integer $N$ and let $t_{ \pm N} \in \mathfrak{G}_{-\varphi \pm N \delta} \subset \mathfrak{n}_{J}$ be nonzero elements. Then $x_{ \pm N}=\left[t_{ \pm N}, d_{r_{0}}\right] \neq 0$. Moreover, $\left[x_{N}, x_{-N}\right] \neq 0$ and we have

$$
\begin{gathered}
t_{ \pm N} v=t_{ \pm N} d_{r_{0}}\left(v_{r_{0}} \otimes w_{r_{0}}\right)+t_{ \pm N} \sum_{r \in R, r \neq r_{0}} d_{r}\left(v_{r} \otimes w_{r}\right)= \\
=x_{ \pm N}\left(v_{r_{0}} \otimes w_{r_{0}}\right)+\sum_{r \in R, r \neq r_{0}}\left[t_{ \pm N}, d_{r}\right]\left(v_{r} \otimes w_{r}\right) .
\end{gathered}
$$

Applying Lemma 3 we conclude that at least one of $t_{ \pm N} v$ is not zero. Since $h t_{J}\left(t_{ \pm N} v\right)=0$ the corollary is proved.

We can now prove Theorem 2 . 
4.1. Proof of Theorem 2, Let $v \in T_{J}(U)$ be a nonzero weight element and ht ${ }_{J}(v)=s \geq$ 1. Consider a $\mathfrak{G}$-submodule $\mathcal{N}$ of $M_{J}(U)$ generated by $v, \mathcal{N}=U(\mathfrak{G}) v$. Since $\mathfrak{n}_{J} v=0$ we have that $\mathcal{N}$ is a proper submodule of $M_{J}(U)$ and all its weight elements have $\mathrm{ht}_{J}$ greater or equal than $s$. But this is a contradiction since by Lemma 1, Corollary 2 and Corollary 1 we can always find $u \in(\mathfrak{G})$ such that $u v \neq 0$ and ht $t_{J}(u v)=\mathrm{ht}_{J}(v)-1$. Therefore ht ${ }_{J}(v)$ must be zero and $T_{J}(U)=M_{J}^{t}(U)$.

Applying Theorem 2 we have

Corollary 3. If $U$ is an irreducible admissible tensor module in $\widetilde{W}_{\mathfrak{l}}$ then the induced module $M_{J}(U)$ is irreducible.

Corollary 3 immediately implies Theorem 1 which provides a powerful tool to construct new irreducible representations for affine Lie algebras by inducing from irreducible tensor modules from $\widetilde{W}_{\mathfrak{l}}$. We conclude with the following observation.

Remark 3. It would be interesting to see if Theorem 2 extends to any admissible tensor module $U$ in $\widetilde{W}_{\mathfrak{l}}$. This would lead to an equivalence of certain subcategories of $\mathfrak{G}$-modules and $\mathfrak{l}_{J}$-modules. Another problem is to check if admissible tensor modules exhaust all irreducible modules in $\widetilde{W}_{\mathfrak{l}}$.

\section{ACKNOWLEDGMENT}

V.F. is supported in part by the CNPq grant (301320/2013-6) and by the Fapesp grant (2014/09310-5). I.K. is supported by the CNPq grant (309742/2013-7) and by the Fapesp grant (2016/08740-1). The authors are grateful to the referee for useful remarks.

\section{REFERENCES}

[BBFK] V.Bekkert, G.Benkart, V.Futorny, I.Kashuba, New Irreducible Modules for Heisenberg and affine Lie Algebras. J. Algebra 373 (2013), 284-298.

[Bu] T.Bunke, Classification of irreducible non-dense modules for $A_{2}^{(2)}$, Algebra Discrete Math., 2 (2009), 11-26.

[C1] B.Cox, Verma modules induced from nonstandard Borel subalgebras, Pacific J. Math. 165 (1994), 269-294.

[DG] I. Dimitrov and D. Grantcharov, Simple weight modules of affine Lie algebras. arXiv:0910.0688.

[DFG] I. Dimitrov, V.Futorny, D.Grantcharov, Parabolic sets of roots, Contemporary Mathematics, 499, (2009), 61-73.

[F1] V.Futorny, Imaginary Verma modules for affine Lie algebras, Canad. Math. Bull., v. 37(2), 1994, 213-218.

[F2] V.Futorny, Representations of affine Lie algebras, Queen's Papers in Pure and Applied Math., v. 106 (1997), Kingston, Ont., Canada.

[F3] V.Futorny, The parabolic subsets of root systems and corresponding representations of affine Lie algebras, Contemporary Math., 131 (1992), part 2, 45-52.

[F4] V.Futorny, Irreducible non-dense $A_{1}^{(1)}$-modules, Pacific J. of Math. 172 (1996), 83-99.

[FK1] V. Futorny, I.Kashuba, Induced modules for Kac-Moody Lie algebras, SIGMA - Symmetry, Integrability and Geometry: Methods and Applications 5 (2009), Paper 026.

[FK2] V.Futorny, I. Kashuba, Generalized loop modules for affine Kac-Moody algebras, Developments in Mathematics. 1ed.: Springer International Publishing, 2014, v. 496, p. 175-183. 
[FS] V.Futorny and H.Saifi, Modules of Verma type and new irreducible representations for affine Lie Algebras, CMS Conference Proceedings, v.14 (1993), 185-191.

[FT] V.Futorny, A.Tsylke, Classification of irreducible nonzero level modules with finite-dimensional weight spaces for affine Lie algebras, J. Algebra 238 (2001), 426-441.

[JK1] H.Jakobsen and V.Kac, A new class of unitarizable highest weight representations of infinite dimensional Lie algebras, Lecture Notes in Physics 226 (1985), Springer-Verlag, 1-20.

[JK2] H.Jakobsen and V.Kac, A new class of unitarizable highest weight representations of infinite dimensional Lie algebras, J. Funct. Anal. 82 (1989), 69-90.

[K] V.Kac, Infinite dimensional Lie algebras, Cambridge University Press, third edition, 1990. Funkts. anal. i ego prilozhen. 21 (1987), 84-85.

[MP] B.Moody, A.Pianzola, Lie Algebras with triangular decomposition, J.Wiley, 1994.

Institute of Mathematics, University of São Paulo, Caixa Postal 66281 CeP 05314-970, SÃo PaUlo, Brazil

E-mail address: futorny@ime.usp.br

E-mail address: kashuba@ime.usp.br 